\title{
Alternative technique for changing from nasal to oral endotracheal tube for orthognathic and nasal surgery by using an airway exchange catheter -a case report-
}

\author{
Seung Hoon Lee ${ }^{1}$, Jung Eun Kim ${ }^{1}$, and Jong-Man Kang ${ }^{2}$ \\ Department of Anesthesiology and Pain Medicine, ${ }^{1}$ Kyung-Hee University Hospital, ${ }^{2}$ Kyung-Hee University Hospital at Gangdong, \\ Kyung-Hee University, Soeul, Korea
}

A 28-year-old male patient with right maxillar, zygomatic arch, orbital wall, and nasal bone fractures had an orthognathic and nasal surgery. Naso-endotracheal intubation is the first choice during surgical correction of dentofacial deformities in an orthognathic surgery; however, its presence can interfere with concomitant surgical procedures on the nose. Traditionally, the naso-endotracheal tube will be removed and replaced with an oro-endotracheal tube. We changed the endotracheal tube from nasal to oral by using an airway exchange catheter. (Korean J Anesthesiol 2014; 67: 48-51)

Key Words: Airway exchange catheter, Nasal surgery, Naso-endotracheal intubation, Orthognathic surgery.

Orthognathic surgical correction in patients with fractures of the maxilla, zygomatic arch, or orbital wall usually requires naso-endotracheal intubation. If there is a concomitant surgical problem in the nose, such as nasal bone fracture, the presence of a tube in the nose would interfere [1].

Therefore, the endotracheal tube must be switched from nasal to oral route to enable proper nasal surgery technique. The traditional method of nasal to oral tracheal tube change is simply removing the naso-endotracheal tube and replacing it with an oro-endotracheal tube via direct laryngoscopy $[2,3]$. Frustratingly, this approach does not guarantee success of reintubation or even ventilation and poses some other potential risks like damage to the previously repaired area and aspiration of blood.

In the current case, the change of endotracheal tube from nasal to oral route was performed safely with an airway exchange catheter (AEC).

\section{Case Report}

A 28-year-old male patient $(80 \mathrm{~kg}, 170 \mathrm{~cm})$ with right maxillar, zygomatic arch, orbital wall, and nasal bone fractures was scheduled for an open reduction and internal fixation of the

Received: April 30, 2013. Revised: June 14, 2013. Accepted: June 21, 2013.

Corresponding author: Jong-Man Kang, M.D., Ph.D., Department of Anesthesiology and Pain Medicine, Kyung-Hee University Hospital at Gangdong, 892, Dongnam-ro, Gangdong-gu, Seoul 134-090, Korea. Tel: 82-2-440-6193, Fax: 82-2-440-7808, E-mail: jongmankang@gmail.com (C) This is an open-access article distributed under the terms of the Creative Commons Attribution Non-Commercial License (http:// creativecommons.org/licenses/by-nc/3.0/), which permits unrestricted non-commercial use, distribution, and reproduction in any medium, provided the original work is properly cited. 
right zygomatic process, maxillary bone, and inferior orbital wall and closed reduction of a nasal fracture. His airway exam revealed a Mallampati class II with a thyromental distance of $7.0 \mathrm{~cm}$. He was intubated with an ID 7.0 nasal Ring, Adair \& Elwyn (RAE) tube for an orthognathic surgery, and the tube was advanced to $28 \mathrm{~cm}$ and secured to the left nostril with tape. The operation time was about 5 hours, and there were no significant adverse events. To facilitate the subsequent nasal procedures, the endotracheal tube had to be exchanged from a nasal tube to an

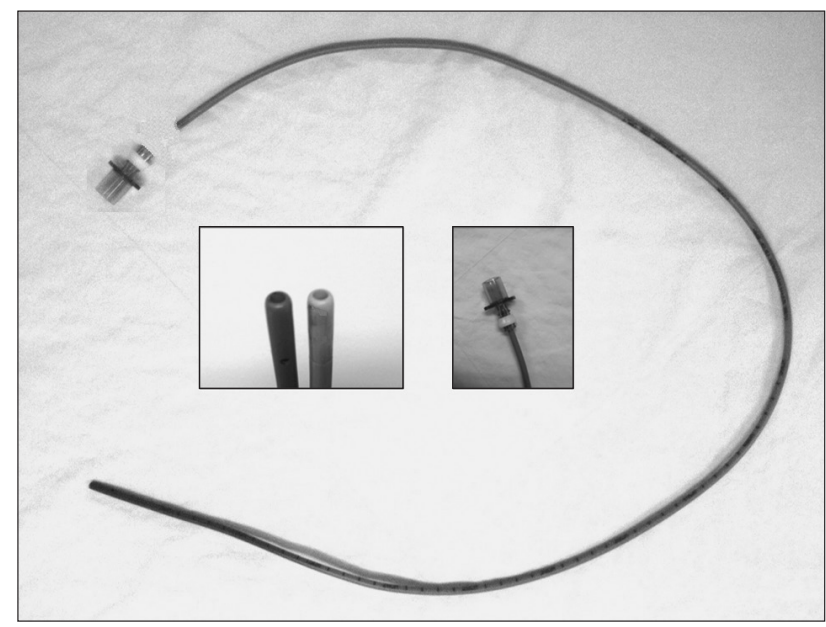

Fig. 1. A 14 Fr. airway exchange catheter (AEC) (Cook airway exchange catheter, Cook Critical Care, Bloomington, IN, USA) with an adaptor (Rapi-Fit ${ }^{\circledR}$ ) fitting the proximal end of AEC. Center, left: Both ends of AEC showing hollowness of the catheter. Center, right: Connection between the proximal end of the AEC and the adapter. oral tube. Oro-endotracheal reintubation with a laryngoscope was expected to be difficult because of severe swelling of the tongue, epiglottis, and retropharyngeal soft tissue. Moreover, the use of a laryngoscope could cause damage to the operation sites with significant pressure to the mandible and maxilla. We decided instead to use a tube exchanger. First, after clearing the airway with suction, we passed a lubricated $14 \mathrm{Fr}$. AEC (Cook airway exchange catheter, Cook Critical Care, Bloomington, IN, USA) through the ID 7.0 nasal RAE tube down to $30 \mathrm{~cm}$ of the AEC at the nostril (Figs. 1 and 2). The nasal RAE tube was mobilized by releasing the tape, and it was removed carefully, leaving the AEC in the patient's nasotracheal airway. A clamp was placed to fix the AEC firmly in the oropharynx at the base of the tongue to prevent accidental movement out of the trachea. Then we pushed the upper end of the AEC posteriorly into the nasopharynx, and at the same time, pulled the AEC above the clamp into and out of the oral cavity with a margil forceps while the clamp stabilized the lower part of the AEC. The upper portion of the AEC was uneventfully delivered from the nasal cavity into the oral cavity. We used a laryngoscope for a light source during the procedure and to keep the mouth cavity open with minimum force to avoid damage to the previously repaired area. Oro-endotracheal intubation with an ID 7.5 oral RAE tube was performed through the AEC, and the tube was secured to the lower lip and chin area with tape. It took less than $2 \mathrm{~min}$ to change the endotracheal tube, and the oxygen saturation remained greater than $97 \%$. Closed reduction of the nasal fracture was then completed, and extubation was performed after confirming the recovery of consciousness and spontaneous breathing.
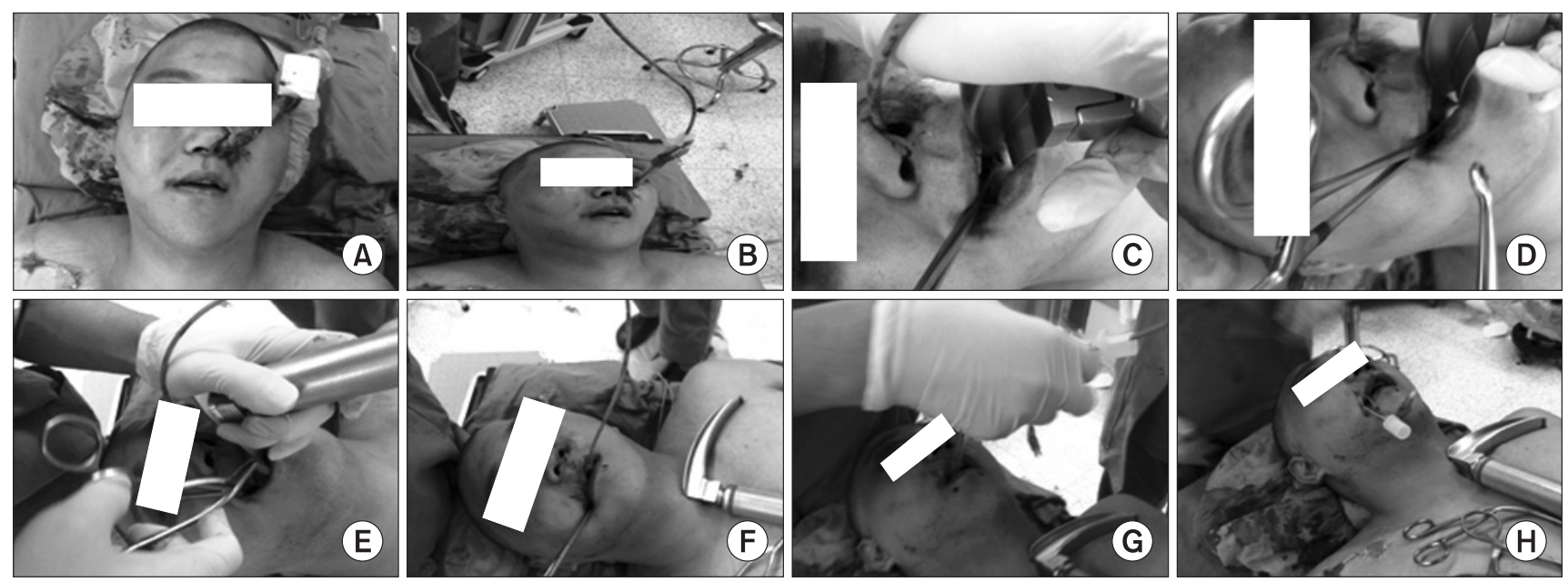

Fig. 2. (A) The nasal Ring, Adair \& Elwyn (RAE) tube in situ. (B) An airway exchange catheter (AEC) is passed through the nasal RAE tube. (C) The nasal RAE tube is removed and a clamp is placed to fix the AEC firmly in the oropharynx. (D, E) The AEC is pulled to deliver the nasal portion into the oral cavity with a margil forceps. (F) The AEC is delivered out of the oral cavity. (G) Oro-endotracheal intubation with a RAE tube is done through the AEC. $(\mathrm{H})$ The oral RAE tube in situ after removal of the AEC. 
Table 1. Airway Exchange Catheters from Cook Medical

\begin{tabular}{lcccc}
\hline \multicolumn{1}{c}{ Name } & Catheter OD (Fr) & Catheter length $(\mathrm{cm})$ & Catheter ID (mm) & For placement of ETT ID (mm) \\
\hline Aintree intubation catheter & 19 & 56 & 4.7 & $\geq 7$ \\
Arndt airway exchange catheter & 14 & 70 & & $\geq 5$ \\
Cook airway exchange catheters & 8 & 45 & 8.6 & $\geq 3$ \\
& 11 & 83 & 3.0 & $\geq 4$ \\
Frova intubating introducers & $14 *$ & 83 & 3.4 & $\geq 7$ \\
& 19 & 35 & 3.6 & $\geq 3$ \\
& 8 & 65 & 3.0 & $\geq 6$ \\
Soft tipped Cook airway exchange catheters & 14 & 65 & 3.0 & $\geq 6$ \\
& 14 & 65 & 2.3 & $\geq 4$ \\
\end{tabular}

The contents of the table are from the website of Cook Medical Incorporatd (Bloomington, IN, USA, www.cookmedical.com). OD: outer diameter, ID: internal diameter, ETT: endotracheal tube, Fr: French. *The catheter used in this case.

\section{Discussion}

The combination of orthognathic and nasal surgery during one operation is common. The naso-endotracheal tube is mostly required for orthognathic surgery but can interfere with nasal surgery, especially rhinoplasty, transnasal surgery, or alar base cinch sutures. The change from a nasal to oral endotracheal tube is usually performed in a stepwise fashion with laryngoscope guidance. The naso-endotracheal tube is extubated, and then laryngoscopic oro-endotracheal intubation is completed. This uninspired method has the potential risks of damage to the previously corrected jaw and intubation failure due to swelling, bleeding, or secretion of a surgical field [2].

Werther et al. [4] converted a naso-endotracheal tube to an oro-endotracheal tube without extubation and reintubation in 10 cases by pushing the nasal portion of the endotracheal tube posteriorly into the nasopharynx and pulling the nasal portion of the tube into and out of the oral cavity. Muto et al. [2] improved the technique using a specially designed retractor and clamp. These techniques carry a potential risk for problems with the pilot balloon assembly, which is replaced and used simultaneously with the same endotracheal tube. Moreover, operations sometimes dictate a change from the nasotracheal tube to another type of tube like a reinforced tube or double lumen tube.

An AEC is a long, small-diameter, hollow, flexible, semirigid catheter. There are various types of AEC, and the proper one should be chosen according to the size of the endotracheal tube (Table 1). It can be introduced through the endotracheal tube before extubation as a stylet for exchange of the tubes or as a conduit for repeated intubation in the intensive care unit. Moreover, it has its own adaptor (Rapi-Fit ${ }^{\circledR}$ ) that can connect the proximal end of the AEC with the ventilator device and operate as a channel to supply oxygen to the patient manually, by insufflation, or by jet ventilation in emergent situations. Therefore, our method of nasal to oral tube changing maybe a safe alternative to other methods even in cases where there is difficulty passing a new endotracheal tube over the AEC. AEC has two potential life-threatening risks as follows: (1) difficulty in passing the new endotracheal tube (ETT) over the AEC, and (2) barotrauma during jet ventilation or even in low-flow insufflation. There are several options to increase success of reintubation. First, one may use laryngoscopy to clear and visualize the supraglottic airway. Second, one may rotate the ETT counterclockwise to $90^{\circ}$ if the tip of the ETT is blocked by the laryngeal inlet. Finally, a small ETT and large AEC may be selected within the recommendations for ETT-AEC combinations. To avoid the devastating complication of barotrauma, one may observe the chest of the patient closely, keep the patient's airway maximally patent to optimize exhalation, and avoid advancing the tip of the AEC past the carina of the trachea [5].

In conclusion, we demonstrated the successful exchange of a nasal to oral endotracheal tube using an AEC.

\section{References}

1. Cottrell DA, Wolford LM. Factors influencing combined orthognathic and rhinoplastic surgery. Int J Adult Orthodon Orthognath Surg 1993; 8: 265-76.

2. Muto T, Akizuki K, Wolford LM. Simplified technique to change the endotracheal tube from nasal to oral to facilitate orthognathic and nasal surgery. J Oral Maxillofac Surg 2006; 64: 1310-2. 
3. Guymon M, Crosby DR, Wolford LM. The alar base cinch suture to control nasal width in maxillary osteotomies. Int J Adult Orthodon Orthognath Surg 1988; 3: 89-95.

4. Werther JR, Richardson G, McIlwain MR. Nasal tube switch: converting from a nasal to an oral endotracheal tube without extubation. J Oral Maxillofac Surg 1994; 52: 994-6.

5. Benumof JL. Airway exchange catheters: simple concept, potentially great danger. Anesthesiology 1999; 91: 342-4. 